\title{
Study of Power Options for Jupiter and Outer Planet Missions
}

\author{
Geoffrey A. Landis and James Fincannon \\ NASA Glenn Research Center, 21000 Brookpark Road, Cleveland OH 44135
}

\begin{abstract}
Power for missions to Jupiter and beyond presents a challenging goal for photovoltaic power systems, but NASA missions including Juno and the upcoming Europa Clipper mission have shown that it is possible to operate solar arrays at Jupiter. This work analyzes photovoltaic technologies for use in Jupiter and outer planet missions, including both conventional arrays, as well as analyzing the advantages of advanced solar cells, concentrator arrays, and thin film technologies.

Index Terms - space exploration, spacecraft solar arrays, solar electric propulsion, photovoltaic cells, concentrator, Fresnel lens, Jupiter missions, outer planets.
\end{abstract}

\section{INTRODUCTION}

Power for missions to Jupiter and beyond presents a challenging goal for photovoltaic power systems [1,2], but NASA missions including the Dawn mission to the asteroid belt and the Juno mission to Jupiter (Figure 1) have shown that it is possible to operate solar arrays beyond Mars, and even at Jupiter, nearly 800 million kilometers from the sun.

Jupiter probes operate in a low sunlight environment, with solar intensity of 3.4 to 4.1 percent that of Earth (varying slightly between aphelion and perihelion of Jupiter's orbit). Simultaneously, the arrays operate at an extreme of low temperatures. The flat-plate equilibrium temperature $\left(\mathrm{T}_{\text {eq }}\right)$ can be calculated from the Stefan-Boltzmann equation,

$$
T_{e q}=\left[\frac{(\alpha-\eta) \mathrm{I}}{\left(\epsilon_{f}+\epsilon_{b}\right) \sigma}\right]^{1 / 4}
$$

where $\alpha$ is the solar absorptivity, I the solar intensity, $\varepsilon$ the front and back thermal emissivity (assuming two-sided emission), and $\sigma$ the Stefan-Boltzmann constant, $5.6710^{-8}$ $\mathrm{W} / \mathrm{m}^{2} \mathrm{~K}^{4}$. Table 1 shows the solar intensity at Jupiter and Saturn, along with the calculated equilibrium temperature, for an assumed absorptivity and emissivity typical of currentgeneration high-efficiency cells.

\section{TABLE 1: THE SOLAR INTENSITY AT JUPITER AND SATURN, AND THE EQUILIBRIUM FLAT-PLATE TEMPERATURE OF A SOLAR ARRAY}

\begin{tabular}{|l|c|c|c|c|c|}
\hline Conditions & $\begin{array}{c}\text { Distance } \\
(\mathrm{AU})\end{array}$ & $\begin{array}{c}\mathrm{I} \\
(\text { suns })\end{array}$ & $\begin{array}{c}\mathrm{I} \\
\left(\mathrm{W} / \mathrm{m}^{2}\right)\end{array}$ & \multicolumn{2}{|c|}{$\mathrm{T}_{\text {eq }}$} \\
\hline Jupiter & $\left({ }^{\circ} \mathrm{C}\right)$ \\
\hline Aphelion & 5.458 & 0.03357 & 46.0 & 133 & -139 \\
\hline Perihelion & 4.950 & 0.04081 & 55.8 & 140 & -133 \\
\hline Saturn \\
\hline Aphelion & 10.12 & 0.0098 & 13.4 & 98 & -175 \\
\hline Perihelion & 9.048 & 0.0122 & 16.7 & 103 & -161 \\
\hline
\end{tabular}

Temperatures calculated for absorptivity 0.92 ; front and back side thermal emissivity of 0.85 , and cell efficiency of $25 \%$.

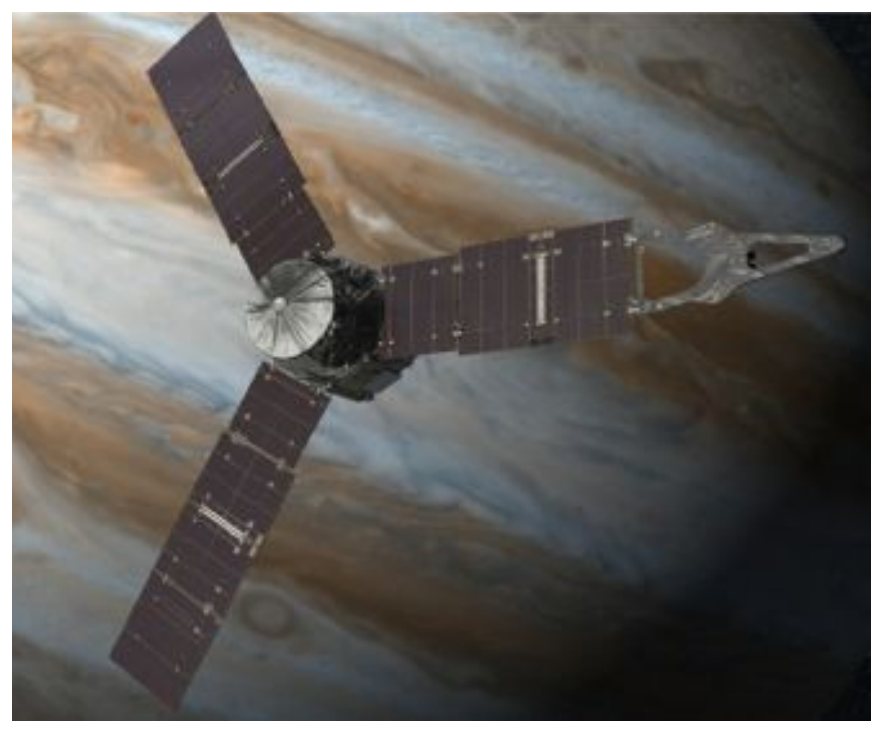

Fig. 1. The Juno probe, currently on its way to Jupiter, features the largest solar array flown by NASA on a mission other than the ISS. Image credit: NASA/JPL/Caltech.

Even without the effect on efficiency, the low solar intensity means that an array at Jupiter must, at aphelion, have thirty times the area of an array in Earth orbit to produce the same power. The reduced conversion efficiencies exacerbate this problem even further.

The low incident solar intensity decreases the voltage, and hence lowers the conversion efficiency of solar cells; while the low operating temperature improves the voltage, and hence increases the efficiency. In addition, operation under the combination of low intensity and low temperature, or "LILT" conditions, can produces anomalous degradation of performance known as LILT effect [3]-[6]. These LILT effects are only partially understood for new generation technologies, but can to some extent be mitigated by screening cells under LILT conditions to select only cells that do not show the anomalous degradation. Such screening was done for the Dawn [3] and Juno [7] missions to the outer solar system.

Except for orbits far from Jupiter, cells for Jupiter missions also experience a high radiation environment. In particular, the inner moons Io and Europa orbit within the Jupiter radiation belts. The Juno mission was designed for a polar observation of Jupiter, and a highly-eccentric polar orbit (Figure 2) was chosen to minimize exposure to the Jupiter radiation belt [8]. However, missions designed to probe the moons must necessarily spend time exposed to high levels of radiation.

Combined with the need for minimum weight, long lifetime, and minimum impact on spacecraft operations, these 
environmental constraints put constraints on the photovoltaic array technology.

\section{RADIATION ENVIRONMENT OF JUPITER}

Many of the most interesting science targets in the Jupiter system are in or near the radiation belts. Jupiter's moon Europa is of particular scientific interest, a moon with an ocean of liquid water hidden below an icy shell. Europa is in a nearly-equatorial orbit, like all of the larger moons of Jupiter, and orbits within the radiation belts, although well outside the peak radiation flux shown in figure 3 .

Proposals for investigating Europa included using icepenetrating radar to determine the thickness of the ice layers covering the ocean, and, for longer term missions, the possibility of landing on the surface and possibly penetrating the ice with a cryobot to investigate the ocean below. Initial concepts for a radar mission proposed orbiting Europa [9]. A new mission to Europa, the upcoming Europa Clipper mission, will not directly orbit Europa, but will be in an eccentric orbit around Jupiter with multiple passes near the moon $[10,11]$. This mission is baselined to be solar powered.

Another application proposed for large solar arrays at Jupiter has been for powering solar electric propulsion (SEP) systems, both for propulsion to Jupiter, arrival at Jupiter, and tours with within the Jupiter system [12]. Such a mission may also require operating solar arrays within the radiation belts.

Calculations were made of the radiation-induced degradation of solar arrays at Jupiter using Jupiter radiation belt models [13] in the Space Environment Information System (SPENVIS) tool $[14,15]$, incorporating the earlier JPL radiation models [16,17]. Figure 3 shows the calculated equivalent radiation fluence per year (expressed in terms of equivalent $1-\mathrm{MeV}$ electron fluence) at various distances from Jupiter, for solar cells with various thicknesses of coverglass (assumed to be identical shielding on front and back side).

Table 2 shows the calculated equivalent radiation fluence and the estimated cell degradation for a one-year mission orbiting at the distance of Europa. Radiation damage also interacts with the LILT degradation, making the LILT problem more severe [6]; this interaction has not been incorporated into this calculation. The amount of radiation degradation depends on the thickness of the coverglass. 0.25 $\mathrm{mm}$ (10 mils) is typical of coverglass thickness for conventional solar arrays used in Earth orbit; for this thin shielding, the radiation fluence at one year is $210^{16} \mathrm{e}^{-} / \mathrm{cm}^{2}$, outside the range of the data set used for calculating degradation. This fluence would be unacceptable for a mission. A thickness of $0.5 \mathrm{~mm}$ (20 mils) might be more typical of the thinnest practical amount of shielding for planar arrays, roughly twice the thickness of conventional solar arrays used in Earth orbit conditions. The thickest coverglass analyzed, $6.3 \mathrm{~mm}$ (250 mils), would be impractical for a planar array but would be reasonable shielding for a concentrator system. Shielding is required on both the front and back of the array; for this calculation, the shielding was assumed to be identical on both sides.

\section{CONCENTRATION APPROACHES}

A number of solar concentration technologies are considered, including both low and high-concentration options. Concentration systems can be categorized as lowconcentration systems, such as mirror trough concentrators, with concentration ratio typically $\leq 2$, and high-concentration systems, using lenses or mirrors.

Low concentration systems have included trough concentrators used in the STEX spacecraft and on early models of Boeing 702 series communications satellites, with a concentration ratio of about 1.5 to 1.7 . The 702 concentrators had significant degradation due to contamination issues, an issue that has been analyzed and is now believed to be understood [18]. Next-generation trough concentrator system includes "CellSaver" system, with roughly a $2 \mathrm{x}$ concentration $[18,19]$.

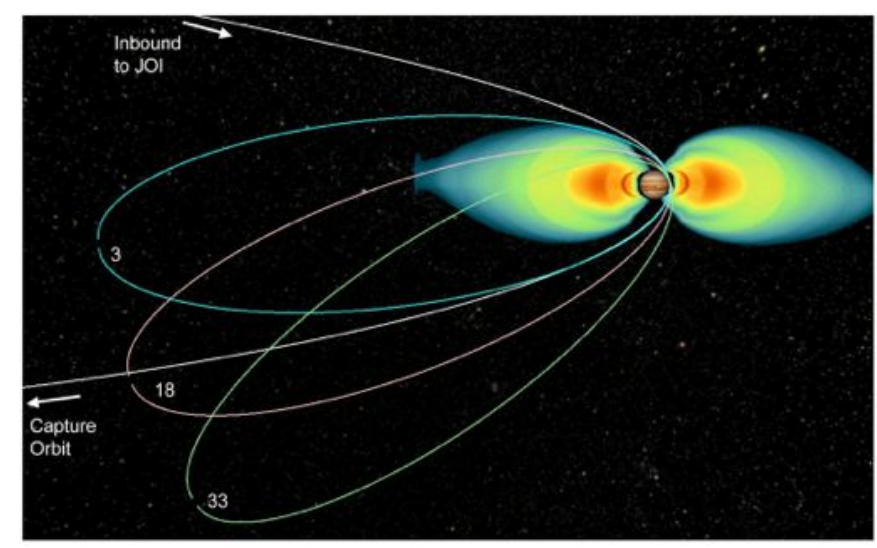

Fig. 2. The orbit of the Juno mission, compared to Jupiter's radiation belts. Image credit: NASA/JPL/Caltech /Institute for Aeronautics and Astronautics.

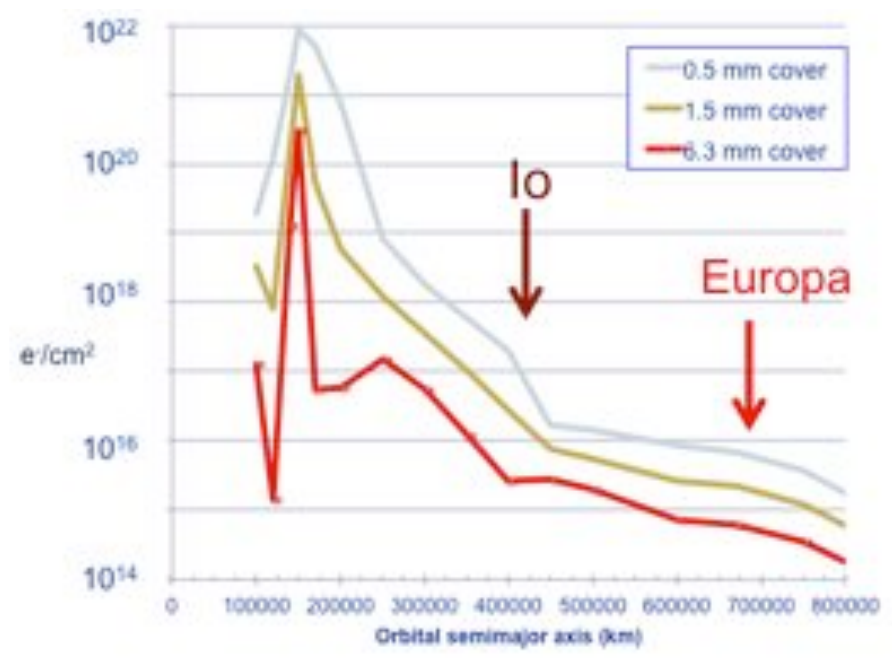

Fig. 3. Calculated radiation fluence per year for an array in equatorial circular orbit around Jupiter, as a function of semimajor axis (expressed as equivalent $1-\mathrm{MeV}$ electron fluence), for several thicknesses of shielding. The orbital distance of moons Io and Europa are marked. 
TABLE 2: EQUIVALENT ONE-YEAR RADIATION DOSE IN JUPITER ORBIT

\begin{tabular}{|l|c|l|}
\hline $\begin{array}{c}\text { Shielding } \\
\text { (coverglass) } \\
\text { thickness }\end{array}$ & $\begin{array}{c}\text { Fluence }\left(\mathrm{e}^{-} / \mathrm{cm}^{2}\right. \\
\text { per year })\end{array}$ & $\mathrm{P} / \mathrm{P}_{\mathrm{o}}$ \\
\hline $0.25 \mathrm{~mm}$ & $20.010^{15}$ & * \\
\hline $0.5 \mathrm{~mm}$ & $9.010^{15}$ & 0.67 \\
\hline $1.5 \mathrm{~mm}$ & $3.610^{15}$ & 0.76 \\
\hline $6.3 \mathrm{~mm}$ & $1.310^{15}$ & 0.84 \\
\hline
\end{tabular}

$0^{\circ}$ inclination, semimajor axis $=671100 \mathrm{~km}$ (Europa distance); $\mathrm{P}_{\max }$ DENI fluence, triple-junction cell (electron/proton damage ratio $=612$ for $\mathrm{P}_{\max }$ ).

*: degradation data not available for fluence of $2 \mathrm{E} 16 \mathrm{e}^{-} / \mathrm{cm}^{2}$

Although low-concentration systems can improve cost somewhat, they are not sufficient to bring the cells out of the LILT condition. High concentration technologies can bring the cells out of the low intensity regime, mitigating or eliminating the LILT effect. To produce the intensity of 1 sun on the cells requires a concentration ratio of 25 to 30 . This concentration ratio is easily achieved by Fresnel lens concentration systems such as the DS-1 array, but is difficult to achieve with trough approaches. Depending on the thermal approach, concentrator approaches can also operate at higher temperatures, although analysis shows that, in the absence of low intensity, low temperature is not a problem. The net result is that improved efficiency due to lower temperatures adds to the array performance, while avoiding the low intensity avoids the reduction in open-circuit voltage and the LILT effect.

Concentrator systems also can be designed with improved radiation tolerance, since a relatively thick glass cover can be put on the photovoltaic element, with only a low effect on mass.

The cost advantage of concentration approaches may also be important for arrays operating at Jupiter. Since power density of the arrays is less by a factor of 25 (or more), using concentration to reduce the area of the array that is covered by solar cells has a much more significant effect at Jupiter than for Earth orbit applications.

However, concentration arrays for space use are at a significantly lower level of technology readiness. To date, the only high concentrator solar arrays flows as primary power in space have been the SCARLET linear-focus Fresnel concentrator (shown in figure 4) flown on the Deep Space 1 ("DS-1") spacecraft, launched in 1998, with a concentration ratio of $7.5[20]$.

Since SCARLET, further development of solar concentrators for space has occurred [21,22], including an evolved version of the SCARLET system, the Stretched Lens Array (SLA) [23]. A demonstration unit of this concentrator is shown in Figure 5 [24]. The SLA is designed to be folded into a flat package for launch, and then stretched out into its deployed configuration on orbit.

A test coupon of the SLA was flown on the TacSat-4 mission in 2011 [25]. Although the test mission demonstrated engineering difficulties, in the deterioration of the lens due to tearing, it also demonstrated the ability of concentration approaches to achieve radiation tolerance due thicker radiation shielding. A new strengthened material has now been developed to avoid this failure mode $[22,25]$.

The SLA concept has been proposed for large area arrays [27].

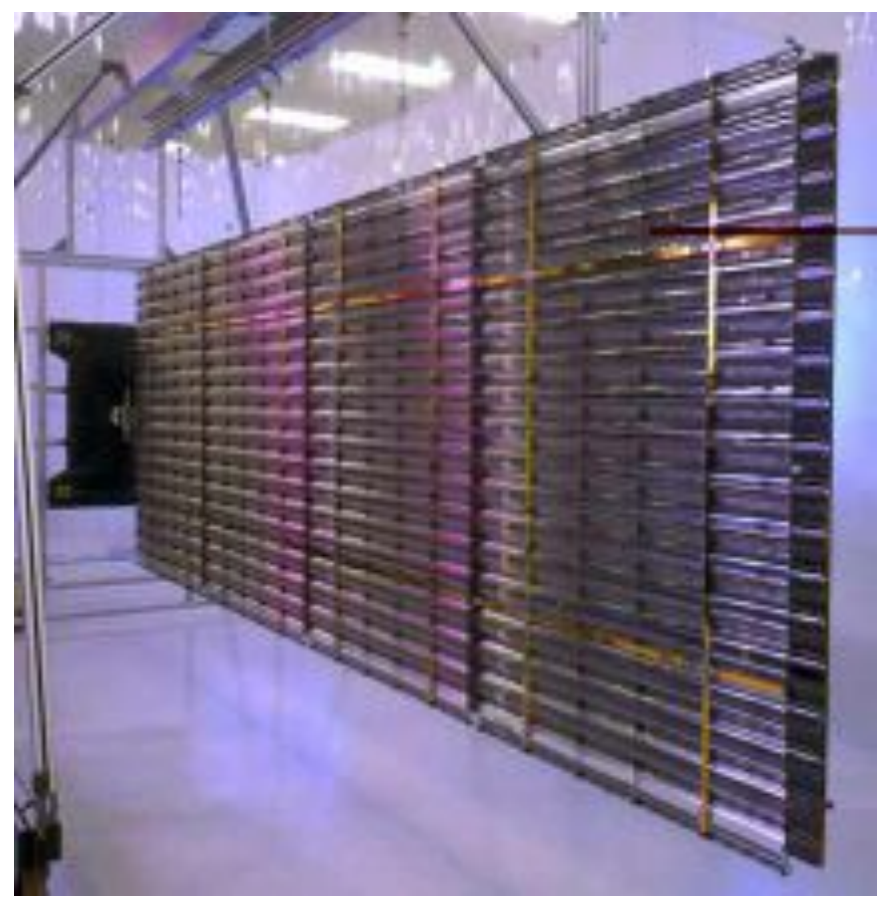

Fig. 4. "SCARLET" linear Fresnel concentrator solar array used on the NASA Deep-Space 1 mission [19].

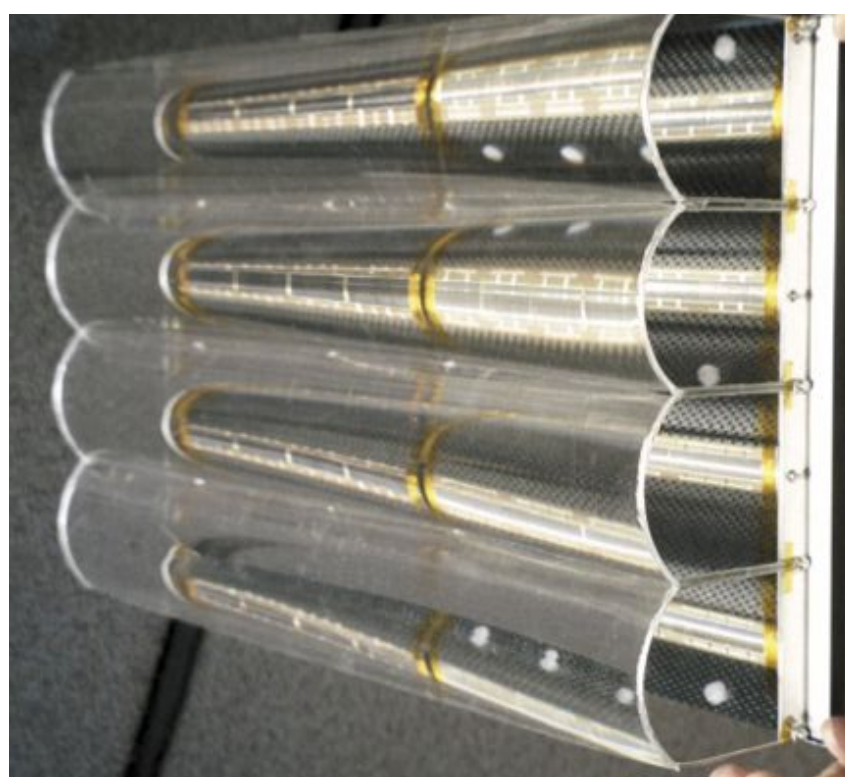

Fig. 5. Demonstration unit of Stretched-Lens Array, a line-focus concentrator system [23]. 
The use of solar concentration, however, comes at a cost. Concentrators add complexity to a mission in terms of more stringent sun-pointing constraints, and hence require a stiffer array structure to limit off-pointing due to array flexibility.

A line-focus concentrator is sensitive to off-pointing in one direction, around an axis parallel to the lens direction. In this direction, the tolerance to off-pointing is inversely proportional to the concentration ratio.

For a concentrator without a refractive secondary, the theoretical off-pointing tolerance is:

$$
\sin \theta \leq 1 / \mathrm{CR}
$$

where $\theta$ is the acceptance half-angle, defined from direct sun pointing to the maximum off-pointing, and $\mathrm{CR}$ the concentration ratio. Thus, for a $25 \mathrm{x}$ concentrator system, which would put an incident intensity at Jupiter of about 1 sun on the focal plane, the theoretical off-pointing tolerance would be \pm 2.3 degrees. Adding a refractive secondary can improve this pointing tolerance. Real-world concentration systems do not achieve the ideal performance, and the allowable acceptance angle is always somewhat less, typically about $1^{\circ}$. This is well within the pointing capabilities of conventional attitude control systems. A more difficult concern is the possibility of losing power due to array flexing.

The axis of the concentrator can be aligned to be perpendicular to direction in which the arrays are most mechanically stiff, to minimize the flexing in the sensitive direction. During most of the mission, the spacecraft will not be subject to any disturbance torques, and it is expected that any flexing of the arrays that result in off-pointing will be quickly damped. For short periods during some science operations, such as close approaches to target moons, the spacecraft may be required to slew. If the required slew rate is fast enough to put torque on the solar arrays sufficient to bend the arrays beyond the tolerated acceptance angle, the arrays may temporarily lose power due to flex-induced off-pointing. An energy storage system would be required to supplement power for a period until such disturbances have been damped out.

The net calculation, incorporating intensity, LILT, and radiation effects, suggests that for a 1-year mission at Europa, concentrator systems at a concentration of $\sim 25$ could produce on the order of $50 \%$ higher end-of-life power for the same array mass. For mission further into the radiation belts (e.g., Io), or longer assumed lifetimes, the advantage increases. Whether this increase in power is worth the added complexity and pointing requirements of a concentrator system is a question for the spacecraft systems engineer.

\section{SATURN AND BEYOND}

Beyond Jupiter, use of solar arrays becomes increasingly difficult. As shown in table 1, at the distance of Saturn, the solar intensity has decreased to $1 \%$ of the intensity at Earth orbit, and the equilibrium temperature of a flat plate solar array is about $-170^{\circ} \mathrm{C}$.

One approach to producing solar power at such large distances from the sun is to use large-aperture concentrators, in which a single large concentrator is used to focus light onto a large solar array placed at the focal plane, rather than multiple small optical elements focusing onto individual cells. This approach is not usable in concentrators on Earth or in near-Earth space, because in the absence of cooling, the concentrated sunlight would produce unacceptable heating at the focal plane. However, at Saturn, a concentration factor of $100 x$ would only raise an array at the focal plane to a temperature equivalent to that experienced on an array at Earth orbit.

In the Inflatable Antenna Experiment in 1996, a 14-meter diameter offset-parabolic reflector was successfully deployed in space $[28,29]$, as shown in figure 6. This technology as demonstrated is adaptable to reflector diameters as large as 25 meters. At Saturn's average distance from the sun, assuming a concentration factor of 100x and a 33\% efficient (AM0, 1-sun) solar array, a reflector of this diameter could produce about $2.4 \mathrm{~kW}$ of power.

\section{CONCLUSIONS}

Exploration of Jupiter and beyond presents significant challenges to solar power systems, due to the extremely low solar intensity and the low operating temperatures of the solar arrays. Many of the most interesting science targets in the Jupiter system are in or near the radiation belts, which presents additional problems.

One approach to addressing the difficulty of solar array operation at Jupiter and beyond is to use solar concentration.

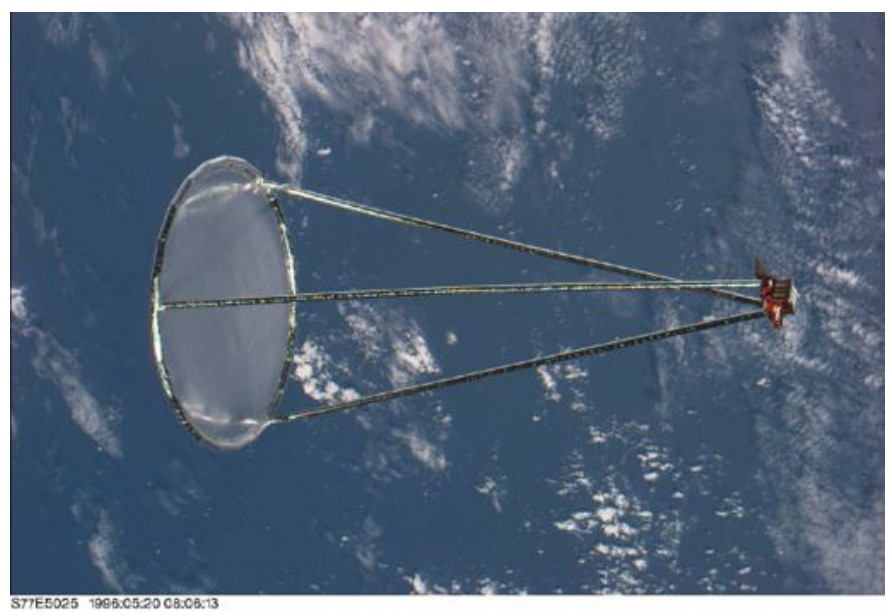

Fig. 6. Inflatable Antenna Experiment, a 14-meter diameter reflector demonstrated in orbit in 1996.

\section{REFERENCES}

[1] M. F. Piszczor, et al., "Advanced Solar Cell and Array Technology for NASA Deep Space Missions," 33rd IEEE Photovoltaic Specialists Conference, pp. 513-517, San Diego, CA, May 11-16, 2008.

[2] J. Fincannon, "Radioisotope Reduction Using Solar Power for Outer Planetary Missions," paper AIAA 2008-5769, 6th International Energy Conversion Engineering Conference, Cleveland, OH, July 28-30, 2008. 
[3] N. S. Fatemi, et al., "Performance of high-efficiency advanced triple-junction solar panels for the LILT Mission Dawn" 31st IEEE Photovoltaic Specialists Conference, Lake Buena Vista, FL, Jan. 3-7, 2005.

[4] P. Stella, R. Mueller, G. Davis, and S. Distefano, "The Environmental Performance at Low Intensity, Low Temperature (LILT) of High Efficiency Triple Junction Solar Cells", paper AIAA-2004-5579, 2nd International Energy Conversion Engineering Conference, Providence, RI, Aug. 16-19, 2004.

[5] D. A. Scheiman and D. B. Snyder, "Low Intensity Low Temperature (LILT) measurements of state-of-the-art triple junction solar cells for space missions," 33rd IEEE Photovoltaic Specialists Conference, San Diego, CA, May 11-16, 2008. doi: 10.1109/PVSC.2008.4922707

[6] R. D. Harris, et al., "In Situ Irradiation and Measurement of Triple Junction Solar Cells at Low Intensity, Low Temperature (LILT) Conditions," IEEE Trans. Nuclear Science, Vol. 55, No. 6, p. 3502-3507 (Dec. 2008).

[7] R. Nybakken, "The Juno Mission to Jupiter - A Pre-launch Update," 2011 IEEE Aerospace Conference, Big Sky, MT, March 5-12, 2011.

[8] S. Kayali, W. McAlpine, H. Becker, and L. Scheick, "Juno Radiation Design and Implementation," 2012 IEEE Aerospace Conference, Big Sky MT, March 3-10, 2012.

[9] K. Clark, et al., Jupiter Europa Orbiter Mission Study 2008 Final Report: The NASA Element of the Europa Jupiter System Mission (EJSM), Task Order \#NMO710851, January 30, 2009

[10] T. Magner, et al., "An Exploration of Icy World Habitability: The Europa Clipper," paper IAC-14.A3.5.1, 65th International Astronautical Congress, Toronto, Canada, Sept. 29-Oct. 3, 2014.

[11] A. Eremenko et al., "Europa Clipper spacecraft configuration evolution," 2014 IEEE Aerospace Conference, pp. 1-13, Big Sky, MT, March 1-8, 2014

[12] N. Strange et al., "Solar Electric Propulsion Gravity-Assist Tours For Jupiter Missions," paper AIAA 2012-451, AIAA/AAS Astrodynamics Specialist Conference, Minneapolis, MN, August 13-16, 2012.

[13] D. Heynderickx, et al., "The JOREM Project: Jupiter Environment, Effects and Shielding Prediction Models for SPENVIS," European Planetary Science Congress 2010, EPSC Abstracts Vol. 5, EPSC2010-628, p. 628, Sept. 2010.

[14] ESA, Space Environmental Information System (SPENVIS): https://www.spenvis.oma.be/

[15] M. Kruglanski, et al., "Space Environment Information System (SPENVIS)," European Geosciences Union General Assembly 2009, Vol. 11, p. 7457, Vienna, Austria, April 19-24, 2009.

[16] H. B. Garrett et al., Galileo Interim Radiation Electron Model, Jet Propulsion Laboratory, California Inst. of Technol., Report JPL 03-006, Pasadena, CA, 2003.

[17] I. Jun, H.B. Garrett and R.W. Evans, "High-energy trapped Particle Environments at Jupiter: an update," IEEE Transactions on Nuclear Science, Vol. 52, No. 6, pp. 2281-2286, 2005.
[18] R. K. Jain, Y. N. Wong, J. Kesapradist, G. van Ommering and M. Eskenazi, "New Concentrator Technology Experiment on a Geosynchronous Satellite," 3rd World Conference on Photovoltaic Energy Conversion, Vol. 1, pp. 865-868, Osaka, Japan, May 18, 2003.

[19] M. Eskenazi, "Design, Analysis, and Testing of the CellSaver Concentrator for Spacecraft Solar Arrays," 29th IEEE Photovoltaic Specialists Conference, pp. 935-938, New Orleans, LA, May 19-24, 2002.

[20] M. D. Rayman, P. Varghese, D. H. Lehman and L. L. Livesay, "Results from the Deep Space 1 Technology Validation Mission," Acta Astronautica, Vol. 47, No. 2-9, July-November 2000, pp. 475-487. doi:10.1016/S0094-5765(00)00087-4.

[21] M. J. O'Neill and M. F. Piszczor, "Inflatable Lenses for Space Photovoltaic Concentrator Arrays," 26th IEEE Photovoltaic Specialists Conference, pp. 853-856, Anaheim, CA, Sept. 30Oct. 3, 1997.

[22] M. O'Neill, et al., "Recent Space PV Concentrator Advances: More Robust, Lighter, and Easier to Track," 42nd IEEE Photovoltaic Specialists Conference, New Orleans, LA, June 14-19, 2015.

[23] H. W. Brandhorst, Jr., J. A. Rodiek, S. R. Best, M. S. Crumpler, M. J. O'Neill, H. Lewis, P. McDermott, "Accomplishments and Objectives of the Stretched Lens Array Technology Experiment (SLATE)," 34th IEEE Photovoltaic Specialists Conference, Philadelphia, PA, June 7-12, 2009.

[24] NASA Technology Transfer Program, "Shedding Light on Solar Power," NASA Spinoff 2002: https://spinoff.nasa.gov/spinoff2002/er_7.html

[25] P. Jenkins et al., "Initial Results from the TacSat-4 Solar Cell Experiment," 39th. IEEE Photovoltaic Specialists Conference, pp. 3108-3111, Tampa, FL, June 16-21, 2013.

[26] M. O'Neill, et al., "Development of More Robust Stretched Lens Array (SLA) Technology with Improved Performance Metrics and Significantly Expanded Applications," 23rd Space Photovoltaic Research and Technology, Cleveland $\mathrm{OH}$, October 28-30 2014

[27] M. O'Neill, J. Howell, J. Fikes, L. Lollar, C. Carrington, N. Suzuki, M. Piszczor, D. Hoppe, M. Eskenazi, D. Aiken, M. Fulton, H. Brandhorst, M. Schuller, and A. McDanal, "Stretched Lens Array SquareRigger (SLASR): A New Space Array for High-Power Missions," IEEE 4th World Conference on Photovoltaic Energy Conversion, vol. 2., pp. 2006-2009, Waikoloa, HI, May 7-12, 2006.

[28] R. E. Freeland and G. Veal, "Significance of the Inflatable Antenna Experiment Technology," paper AIAA-98-2104, 39th AIAA/ASME/ASCE/AHS/ASC Structures, Structural Dynamics, and Materials Conference and Exhibit, Long Beach, CA, April 20-23, 1998.

[29] R. E. Freeland et al., "Large Inflatable Deployable Antenna Flight Experiment Results," paper IAF-97-1.3.01, 48th International Astronautical Congress, Turin, Italy, October 610, 1997. 